\title{
Parâmetros sanguíneos de cavalos alimentados com concentrados lipídicos submetidos a treinos aeróbicos montados
}

\author{
Horse blood parameters fed lipidic concentrates subjected to aerobic training rider
}

\author{
OLIVEIRA, Kátia de ${ }^{1 *}$; SANTOS, Vanessa Pillon $\operatorname{dos}^{2}$; COSTA, Ciniro ${ }^{1}$; FAUSTINO, \\ Marília Gabriela ${ }^{2}$; SÁ, Janaina Carolina de ${ }^{1}$; HEINRICHS, Reges ${ }^{1}$; MEIRELLES, \\ Paulo Roberto de Lima ${ }^{3}$
}

\author{
${ }^{1}$ Universidade Estadual Paulista, Campus Experimental de Dracena, Departamento de Zootecnia, \\ Dracena, São Paulo, Brasil. \\ ${ }^{2}$ Universidade de Marília, Faculdade de Ciências Agrárias, Marília, São Paulo,Brasil. \\ ${ }^{3}$ Universidade Estadual Paulista, Faculdade de Medicina Veterinária e Zootecnia, Departamento de \\ Melhoramento e de Nutrição Animal, Botucatu, São Paulo, Brasil. \\ *Endereço para correspondência: katia@dracena.unesp.br
}

\section{RESUMO}

Avaliou-se o fornecimento de concentrados com baixo e alto teor de óleo de soja a cavalos atletas, submetidos a duas intensidades de treinos aeróbicos montados, sobre a resposta metabólica de parâmetros bioquímicos do sangue, de importância ao desempenho esportivo. Foram utilizados quatro cavalos, em delineamento experimental quadrado latino, com tratamentos em esquema fatorial $2 \times 2$ (duas inclusões de óleo de soja e duas rotinas de treinos aeróbicos). Os tratamentos foram compostos por teores de 5 e $15 \%$ de óleo de soja nos concentrados e duas intensidades de treinos montados por 40 e $60 \mathrm{~min}$, classificadas como aeróbicas. As amostras de sangue foram colhidas após o último treino de 40 ou 60min, de cada período experimental. Monitorou-se, após o exercício, os parâmetros bioquímicos, triglicerídeos (TG), colesterol total (CT), glicose (GLI) e lactato (LAC). Houve redução no teor TG $(\mathrm{P}<0,05)$ para cavalos consumindo $15 \%$ de óleo e treinados aerobicamente por $60 \mathrm{~min}$., o CT elevou-se em função do aumento da inclusão de óleo $(\mathrm{P}<0,05)$, incremento LAC $(\mathrm{P}<0,05)$ em cavalos treinados por $60 \mathrm{~min}$., independente do nível de óleo ingerido $(1,48 \mathrm{mmol} / \mathrm{L})$, bem como não se verificou efeito $(\mathrm{P}>0,05)$ dos tratamentos sobre GLI. Concluiu-se que, para cavalos atletas em atividade aeróbica, o oferecimento de concentrado com alto teor óleo de soja deve ser associado ao treino montado de maior intensidade.

Palavras-chave: atividade física, desempenho atlético, equino, óleo de soja.

\section{SUMMARY}

The feeding diets were evaluated containing low and high levels of soybean oil for athlete horses submitted to two rider aerobic training intensities, on the metabolic response of the blood biochemical parameters (triglyceride, total cholesterol, glucose and lactate) of importance to sports performance. Four horses were used in latin square design with treatments in a 2x2 factorial arrangement (two levels of soybean oil and two aerobic training intensities rider). Treatments consisted of levels of 5 and $15 \%$ soybean oil concentrates and two aerobic training intensities, 40 and 60 minutes. Blood samples were collected after the last workout, 40 or $60 \mathrm{~min}$ in each experimental period. Plasmatic parameters were monitored, triglyceride (TG), total cholesterol (TC), glucose (GLU) and lactate (LAC) after exercise. There was a reduction in $\mathrm{TG}(\mathrm{P}<0.05)$, for horses consuming $15 \%$ of soybean oil and trained by aerobic for 60 minutes, the CT increased with increasing soybean oil $(\mathrm{P}<0.05)$, increase in LAC $(\mathrm{P}<0.05)$ in trained horses for 60 minutes regardless of level of soybean oil $(1.48 \mathrm{mmol} / \mathrm{L})$ and there was no effect $(\mathrm{P}>0.05)$ of treatment on GLU. It can be concluded that the offer of concentrate with high content of soybean oil, should be associated with higher intensity aerobic training for athletic equine.

Keywords: athletic performance, equine, physical activity, soybean oil. 


\section{INTRODUÇÃO}

O uso de dietas lipídicas na alimentação de cavalos atletas tem demonstrado reduzir os riscos de distúrbios digestivos, como laminite e cólica (RESENDE JÚNIOR et al., 2004), bem como por melhorar o desempenho durante atividade física (RIBEIRO et al., 2009). No metabolismo do animal, a suplementação lipídica aumenta a concentração de glicose sanguínea e retarda o acúmulo de ácido lático, por diminuir a utilização do glicogênio, nos cavalos em treinamento aeróbico (GODOI et al., 2009a,b). Esta informação é de suma importância para o bom desempenho esportivo, pois a fadiga tem relação estreita com acúmulo de lactato (MORGADO \& GALZERANO, 2006).

De acordo com Piccione et al. (2009), a suplementação com óleo tem resultado em queda nos níveis plasmáticos de triglicerídeos (TG), sugerindo aumento na oxidação dos ácidos graxos, no interior do músculo. Ainda, o treinamento tem mostrado produzir aumento na atividade da lipoproteína lipase no músculo de cavalos e causar a preferência no uso de ácidos graxos como fonte de energia. Isto demonstra que o treinamento e $\mathrm{o}$ fornecimento de lipídeos atuam em sinergismo na melhora do desempenho atlético de cavalos (OLDRUITENBORGH-

OOSTERBAAN, 2002).

Contudo, os parâmetros lipídicos e bioquímicos avaliados na experimentação de equinos sofrem grande variabilidade em seus valores devido a fatores, como doenças, a idade, o sexo, os exercícios físicos, a dieta, o desempenho, o manejo, o clima e os métodos laboratoriais usados nas análises (O’CONNOR et al., 2004). A falta de consistência nos resultados observadas nas pesquisas, em relação aos benefícios do uso de óleo na dieta de cavalos atletas, é creditada, não somente, às variações nas determinações dos parâmetros bioquímicos, mas também às diferenças na quantidade e tipo de óleo adicionado, regime de treinamento $\mathrm{e}$ intensidade do exercício-teste em esteira (MATTOS et al., 2006).

As avaliações de desempenho podem ser realizadas em ambiente laboratorial ou a campo. A segunda metodologia apresenta as melhores condições para avaliar o desempenho atlético do cavalo, por demonstrar a real força necessária ao trabalho, pois considera a pista e os pesos da sela e do cavaleiro (EVANS, 2007). Desta forma, objetivou-se avaliar o fornecimento de concentrados com baixo e alto teor de óleo de soja a cavalos atletas, submetidos a duas intensidades de treinos aeróbicos montados, sobre a resposta metabólica de parâmetros bioquímicos do sangue, de importância ao desempenho esportivo.

\section{MATERIAL E MÉTODOS}

O experimento foi realizado no setor de Equideocultura pertencente ao Departamento de Zootecnia, Faculdade de Ciências Agrárias, Universidade de Marília, UNIMAR/SP e na UNESP/Campus Experimental de Dracena. Foram utilizados quatro cavalos castrados, mestiços, com idade aproximada de sete anos e peso vivo (PV) médio de 400kg. O delineamento experimental utilizado foi o quadrado latino $4 \times 4$ (animais $\mathrm{x}$ períodos), com a unidade animal constituída por um cavalo, totalizando quatro repetições (GOBESSO et al., 2011). Os tratamentos consistiram de quatro grupos experimentais, em esquema fatorial $2 \times 2$, contendo dois teores de óleo de soja no concentrado (baixo e alto) e duas 
intensidades de treino aeróbico montado (40 e 60min).

Cada período experimental foi composto por seis semanas, com intervalo de descanso de um mês entre os períodos experimentais (BRAGA et al., 2008). Esta medida foi tomada para não haver confundimento entre as intensidades de treinos montados e os níveis de inclusão de óleo, ou seja, evitando efeito residual do tratamento anterior em relação ao atual durante o rodízio do quadrado latino. De acordo com Stubbs et al. (2011), o período de um mês é suficiente para ocorrer alterações no condicionamento físico de cavalos, sejam estes, de ganho ou perda. Ainda, King et al. (2008), relatam que a concentração sanguínea de ácido graxo cai drasticamente, próximo a $80 \%$, a partir de dez dias de se modificar a suplementação com óleo. Portanto, tomadas estas precauções, fundamentadas nas pesquisas citadas acima, pode-se considerar apropriado o uso do delineamento experimental em quadrado latino, para avaliar acuradamente, o desempenho esportivo de eqüinos atletas. Os cavalos foram condicionados à atividade física em período préexperimental por oito semanas, bem como ao consumo do concentrado com baixo teor de lipídeo. Os exercícios constituíram-se por $15 \mathrm{~min}$ de trote em andador automatizado sem a presença do cavaleiro. Durante os intervalos, entre os períodos experimentais, os animais consumiram a dieta oferecida no período pré-experimental, bem como realizaram a mesma atividade física. Ainda, os animais foram desverminados com antiparasitário de amplo-espectro à base de ivermectina, previamente ao início do ensaio e a cada três meses no decorrer do experimento.

Formularam-se dois concentrados contendo baixa e alta inclusão de lipídeo, ou seja, 5 e $15 \%$ de óleo de soja, de maneira a serem isoenergéticos e isoprotéicos, $3,65 \mathrm{Mcal} / \mathrm{kg}$ e $13,6 \%$ $\mathrm{PB}$, respectivamente. $\mathrm{Na}$ Tabela 1 encontra-se a composição centesimal, protéica e energética dos concentrados experimentais. $\mathrm{O}$ consumo de alimento diário, na $\mathrm{MS}$, foi de $2 \%$ do $\mathrm{PV}$, constituído por $70 \%$ de feno de tifton $(11,26 \% \mathrm{~PB}, 73,02 \%$ de fibra em detergente neutro e $36,63 \%$ de fibra em detergente ácido na base da MS) e 30\% de concentrado (FRAPE, 2008), para atingir as necessidades nutricionais de cavalos em atividade física moderada, seguindo as recomendações do NRC de 2007. O concentrado foi fornecido duas vezes ao dia e a água e o sal mineral oferecidos ad libitum. Os cavalos permaneceram durante o estudo em baias de alvenaria medindo $4 \times 4 \mathrm{~m}$, contendo material de cama no piso, bebedouro automático e comedouros para os fornecimento de concentrado e sal mineral, bem como dispondo de fenil para o fornecimento de volumoso. O concentrado foi oferecido nas duas primeiras refeições do dia, em quantidades iguais, enquanto que $\mathrm{o}$ volumoso foi dividido em três refeições. Desta forma, os arraçoamentos foram realizados às $7 ; 13$ e $19 \mathrm{~h}$.

Os protocolos de exercícios físicos, aos que os cavalos foram submetidos no período experimental, constituíram-se por atividade aeróbica, realizada montada em pista plana de areia, com a frequência de cinco dias/semana e intensidades de 40 ou $60 \mathrm{~min} / \mathrm{sessão,} \mathrm{realizado} \mathrm{pelo} \mathrm{mesmo}$ equitador habilitado. Assim, foram designados dois protocolos de atividade aeróbica constituídos da seguinte forma, $12 \mathrm{~min}$ ao passo e $28 \mathrm{~min}$ ao trote, totalizando $40 \mathrm{~min} /$ treino (PRO-40') e $18 \mathrm{~min}$ ao passo e $42 \mathrm{minu}$ ao trote, resultando em 60 minutos de atividade física/treino (PRO-60'). Segundo o NRC (2007), classifica como trabalho moderado, cavalos realizando atividade 
física de 3 - 5h semanais, contendo $30 \%$ do exercício físico no andamento ao passo. Neste sentido, os protocolos experimentais resultaram em 3 e 5 horas semanais de trabalho montado, respectivamente, para $\mathrm{PRO}-40^{\prime}$ e $\mathrm{PRO}-$
60 ', e $30 \%$ de atividade ao passo para ambos protocolos, caracterizando intensidades de exercícios aeróbicos diferenciados, dentro da mesma categoria de trabalho moderado.

Tabela 1. Composição centesimal e nutricional dos concentrados lipídicos, na base da matéria seca

\begin{tabular}{lcc}
\hline \multirow{2}{*}{ Ingredientes (\%) } & \multicolumn{2}{c}{ Inclusão de Óleo de Soja } \\
\cline { 2 - 3 } & Baixa $-5 \%$ & Alta $-15 \%$ \\
\hline Milho em grão & 80 & 30 \\
Farelo de Soja & 15 & 10 \\
Farelo de Trigo & - & 45 \\
Óleo de Soja Refinado & 05 & 15 \\
Total & 100 & 100 \\
\hline \multicolumn{2}{c}{ Composição Nutricional } \\
\hline Energia Digestível (Mcal/kg)* & 3,6 & 3,7 \\
Proteína Bruta (\%) & 13,4 & 13,8 \\
Fibra em detergente ácido (\%) & 8,5 & 6,7 \\
\hline
\end{tabular}

*Valor calculado de acordo com NRC (2007) para alimentos concentrados.

As variáveis sanguíneas, monitoradas nos cavalos em treinamento aeróbico montado, constituíram-se de parâmetros bioquímicos, triglicerídeos (TG), colesterol total (CT), glicose e lactato. As amostras de sangue foram colhidas após o último treino de 40 ou $60 \mathrm{~min}$, de cada período experimental. Para análise dos lipídeos plasmáticos, as colheitas foram realizadas por meio de punção da veia jugular, com agulhas 40x12, em tubos sem anticoagulante e com capacidade para $10 \mathrm{~mL}$, e estas amostras encaminhadas ao laboratório no prazo máximo de quinze minutos. As concentrações de TG e CT foram determinadas por método colorimétricoenzimático (BERGMEYER, 1974).

Para avaliação sanguínea de glicose e lactato, as amostras de sangue foram coletadas da veia jugular em tubos a vácuo contendo fluoreto de sódio para determinação de glicose e para o lactato uma alíquota de sangue total foi encaminhada ao lactímetro (YSI 2300 STAT Plus ${ }^{\text {TM }}$ Glucose \& Lactate Analyzer. YSI Incorporated, EUA). O sangue foi acondicionado em ambiente refrigerado e centrifugado, o plasma obtido foi armazenado e congelado até o momento da leitura, realizado logo após o treino, utilizando-se de kits bioquímicos de glicemia (Katal) e mensurados em espectrofotômetro. As variáveis sanguíneas bioquímicas foram submetidas à análise de variância do Statistical Analysis System (SAS Institute, 2000) e as comparações entre as médias por meio do teste de Tukey ao nível de significância de 5\%. 


\section{RESULTADOS E DISCUSSÃO}

Observou-se efeito significativo das dietas e treinamentos experimentais sobre a concentração plasmática dos TG, em que houve redução no teor do mesmo $(\mathrm{P}<0,05)$, somente para cavalos consumindo concentrados contendo a inclusão de $15 \%$ de óleo de soja e treinados aerobicamente por $60 \mathrm{~min}$ (Tabela 2). Os dados da literatura são conflitantes quanto aos efeitos da adição de óleo na alimentação de cavalos exercitados, sobre o TG sanguíneo. Há relato concordante a atual pesquisa, como o trabalho de OldruitenborghOosterbaan et al. (2002), em que redução na ordem de $33 \%$ na concentração plasmática do TG em cavalos atletas $(\mathrm{P}<0,05)$, foi encontrado na dieta com $12 \%$ de gordura em comparação ao controle, contendo $1,5 \%$ de lipídeos, sendo esta resposta de mesma magnitude aos resultados encontrados na Tabela 2.

Tabela 2. Valores médios dos parâmetros sanguíneos ( $\mathrm{mmol} / \mathrm{L})$ de cavalos atletas alimentados com concentrados contendo baixa e alta inclusão de óleo de soja e submetidos a dois protocolos de intensidades de treinos aeróbicos montados

\begin{tabular}{|c|c|c|c|c|c|}
\hline \multirow{3}{*}{ Variáveis } & \multicolumn{4}{|c|}{ Inclusão de óleo de soja } & \multirow{3}{*}{$\mathrm{CV}(\%)^{1}$} \\
\hline & \multicolumn{2}{|c|}{ Baixa - 5\% } & \multicolumn{2}{|c|}{ Alta $-15 \%$} & \\
\hline & PRO-40* & PRO-60** & PRO-40 & PRO-60 & \\
\hline Triglicerídeos & $0,30^{\mathrm{a}}$ & $0,29^{\mathrm{a}}$ & $0,27^{\mathrm{a}}$ & $0,19^{b}$ & 19,8 \\
\hline Colesterol Total & $2,28^{\mathrm{a}}$ & $2,45^{\mathrm{a}}$ & $3,01^{\mathrm{b}}$ & $3,40^{\mathrm{b}}$ & 13,7 \\
\hline Glicose & $3,20^{\mathrm{a}}$ & $3,06^{\mathrm{a}}$ & $3,50^{\mathrm{a}}$ & $3,24^{\mathrm{a}}$ & 17,9 \\
\hline Lactato & $0,90^{\mathrm{a}}$ & $1,48^{\mathrm{b}}$ & $0,96^{\mathrm{a}}$ & $1,47^{\mathrm{b}}$ & 6,0 \\
\hline
\end{tabular}

*Protocolo de treinamento aeróbico montado por 40min (PRO-40'); **Protocolo de treinamento aeróbico montado por 60min (PRO-60').

${ }^{\mathrm{a}, \mathrm{b}}$ Médias com letras diferentes na mesma linha diferem entre si pelo teste de Tukey $(\mathrm{P}<0,05)$;

${ }^{1} \mathrm{CV}=$ coeficiente de variação.

Diferentemente, Harkins et al. (1992), trabalhando com eqüinos em atividade física submáxima, verificaram incremento no TG plasmático de animais consumindo dieta com adição de óleo, em que o valor médio observado foi de $0,39 \mathrm{mmol} / \mathrm{L}$. Considera-se que estas divergências entre resultados verificado nos ensaios, deve-se a diferentes condições experimentais como raças, manejo alimentar, intensidade de treino, treinamento a campo ou em esteira, uso de exercício teste, entre outras.

A queda no teor plasmático do $\mathrm{TG}$ em cavalos treinados recebendo concentrados suplementados com óleos pode ser uma resposta secundária ao aumento na atividade da enzima lipoproteína lípase, promotora do fluxo de ácido graxo aos músculos esqueléticos. Assim, o aumento na atividade desta enzima ao nível muscular, pode estar associado ao incremento na capacidade oxidativa dos músculos, tornando-se vantajoso aos cavalos exercitados. De acordo com Geelen et al. (1999), cavalos alimentados com dietas lipídicas aumentam a captação de ácidos graxos pelo músculo, incrementando o uso destes compostos como combustível no metabolismo aeróbio, e portanto, facilitando a oxidação dos ácidos graxos durante o exercício. Ainda, mudanças metabólicas similares são induzidas pelo exercício. 
Acredita-se que a atividade física promova adaptações específicas no metabolismo lipídico, que facilitam a utilização de ácidos graxos eficientemente, quando a demanda energética aumenta, como verificado na atual pesquisa em que $o$ efeito significativo sobre o parâmetro TG foi observado nos cavalos alimentados com maior teor de óleo e submetidos ao maior trabalho aeróbico (PRO-60').

A concentração plasmática do CT de cavalos treinados aerobicamente foi significantemente elevada em função do aumento do teor de óleo experimental (Tabela 2), monitorados após o exercício. $\mathrm{O}$ aumento na concentração sanguínea do $\mathrm{CT}$ tem sido relatado às dietas suplementadas com lipídeos para cavalos atletas, com níveis de adição até $12 \%$ de óleo no concentrado, cujos valores resultantes foram de 3,10 e $3,0 \mathrm{mmol} / \mathrm{L}$, respectivamente aos estudos de Hambleton et al. (1980) e Oldruitenborgh-Oosterbaan et al. (2002), similares aos resultados deste ensaio em que observou-se $3,40 \mathrm{mmol} / \mathrm{L}$. De acordo com Geelen et al. (1999), incremento no conteúdo sanguíneo do colesterol tem sido associado a maior atividade da enzima lipoproteína lípase. A partir disto, torna-se importante a observação, do presente ensaio, relativo ao incremento do CT sanguíneo, pois reforça a teoria sobre a maior atividade da enzima lipoproteína lípase no músculo de equinos alimentados com dietas lipídicas e exercitados aerobicamente, resultando em maior capacidade oxidativa (HALLEBEEK \& BEYNEN, 2002, BRANDI et al., 2008). Ainda, a análise conjunta dos resultados obtidos, no presente ensaio, da ação das dietas experimentais às concentrações plasmáticas dos TG e CT em cavalos exercitados, demonstra que o incremento no teor do CT ocorre também em consequência da redução dos níveis sanguíneos do TG. Neste sentido, pesquisas têm implicado ação da enzima lipoproteína lípase, como responsável por este comportamento de resposta metabólica dos parâmetros lipídicos. Sabe-se que há correlação positiva entre a concentração de $\mathrm{CT}$ e atividade da enzima lipoproteína lípase, devido a liberação de partículas na corrente sanguínea de colesterol e fosfolipídeos quando os TG são hidrolisados pela mesma (GEELEN et al., 1999). Portanto, isto resulta em teores plasmáticos dos TG e CT inversamente proporcionais, como resposta adaptativa metabólica, à inclusão de dietas lipídicas em cavalos atletas.

Ausência de alterações a discretos incrementos na concentração plasmática da glicose (dentro dos valores normais de referência) são verificados nos cavalos após o exercício e alimentados com concentrados lipídicos, devido a diferenças na intensidade e duração da atividade física (OLIVEIRA et al., 2010). $\mathrm{Na}$ atual pesquisa não se observou efeito $(\mathrm{P}>0,05)$ da suplementação com óleo de soja, bem como da duração do treino aeróbico, sobre este parâmetro, no qual o valor médio foi de $4,2 \mathrm{mmol} / \mathrm{L}$. Similarmente, estudos têm encontrado o mesmo tipo de resposta, referente à avaliação da suplementação lipídica em cavalos após o exercício, em que os resultados variaram de 3,4 à $6,9 \mathrm{mmol} / \mathrm{L}$ para inclusões perfazendo até $12 \%$ de óleo nos concentrados (MARQUEZE et al., 2001; OLDRUITENBORGHOOSTERBAAN et al., 2002; RIBEIRO et al., 2004; LINDBERG et al., 2006).

De acordo com Brandi et al. (2009), a normalidade da glicemia verificada em cavalos exercitados é mantida pelo aumento na concentração da enzima glicose-6-fosfato no músculo, que causa redução na retirada da glicose circulante no sangue, sendo esta reação metabólica, uma resposta adaptativa ao incremento 
de lipídeos na alimentação dos animais. Ainda, a adaptação do cavalo ao treinamento influencia sua capacidade em realizar a atividade física, produzindo menor quantidade de glicose para suportar o exercício (GEOR et al., 2002). Isto foi observado na Tabela 2, em que os valores glicêmicos apresentaram-se próximos ao limite inferior do valor de referência de $4-7 \mathrm{mmol} / \mathrm{L}$, sinalizando bom condicionamento físico dos eqüinos ao treinamento aeróbico realizado durante o ensaio. Acredita-se que a adaptação metabólica lipídica e física foi preponderante na manutenção da glicemia normal, porém baixa, observada nos cavalos do presente ensaio.

No presente trabalho houve aumento $(\mathrm{P}<0,05)$ na concentração plasmática de lactato, nos grupos experimentais de cavalos submetidos à atividade aeróbica de maior intensidade (PRO-60'), independente do nível de óleo contido nos concentrados (Tabela 2). Esta resposta concorda com os resultados observados por Lindberg et al. (2006), em que cavalos consumindo concentrado lipídico contendo $9,7 \%$ de óleo, tiveram incremento no teor de lactato sanguíneo para $4 \mathrm{mmol} / \mathrm{L}$ em comparação ao grupo controle recebendo $5,7 \%$ de lipídeos. Entretanto, os valores verificados na literatura para cavalos consumindo óleo e treinados na intensidade submáxima e intensa, foram de 4,5 e $14,7 \mathrm{mmol} / \mathrm{L}$, respectivamente, sendo superiores ao teor de lactato de $1,48 \mathrm{mmol} / \mathrm{L}$ observado na atual pesquisa (HARKINS et al., 1992; HARRIS et al., 1999; MARQUEZE et al., 2001; OLDRUITENBORGHOOSTERBAAN et al., 2002; GOMIDE et al., 2006; WATANABE et al., 2006). Ainda, apesar do incremento significativo no lactato observado com a suplementação de óleo neste ensaio, verificou-se também a ocorrência de valores baixos desta concentração nos diferentes tratamentos, máximo de $1,48 \mathrm{mmol} / \mathrm{L}$ conforme apresentado na Tabela 2, no qual foram inferiores aos valores limítrofes do metabolismo aeróbio $(<2 \mathrm{mmol} / \mathrm{L})$ e da acidose clínica $(2-5 \mathrm{mmol} / \mathrm{L})$. Assim, estes valores reduzidos de lactato verificados nos cavalos, no presente estudo, sugerem que os animais se exercitaram utilizando o metabolismo aeróbio, como principal via produtora de energia, bem como se encontravam em bom condicionamento físico (BRANDI et al., 2010).

A suplementação de óleo em cavalos montados, da atual pesquisa, mostrou desenvolver adaptação metabólica às maiores cargas de lipídeo e de treino, conforme denotado pelo decréscimo na concentração de triglicerídeos sanguíneo. Esta redução plasmática dos TG pode ter acontecido como consequência do aumento da habilidade do músculo exercitado em utilizar ácidos graxos, devido ao incremento na atividade da enzima lipoproteína lípase no músculo. Adicionalmente, todos os cavalos em treinamento montado, consumindo concentrado com adição de óleo, apresentaram valores de lactato similares ao basal, denotando predominância do metabolismo oxidativo. Desta forma, concluiu-se que, para cavalos atletas em atividade aeróbica, o oferecimento de concentrado com alto teor óleo de soja deve ser associado ao treino montado de maior intensidade.

\section{REFERÊNCIAS}

BERGMEYER, H.V. Methods of enzymatic analysis. 2nd ed. New York: Verlag Chemie/Academic Press. 1974. 200 p. 
BRAGA, A.C.; ARAÚJO, K.V.; LEITE, G.G.; MASCARENHAS, A.G. Níveis de fibra em detergente neutro em dietas para eqüinos. Revista Brasileira de Zootecnia, v.37, n.11, p.1965-1972, 2008 .

BRANDI, R.A.; FURTADO, C.E.; MARTINS, E.N.; FREITAS, E.V.; LACERDA-NETO, J.C.; QUEIROZNETO, A. Efeito de dietas com adição de óleo e do treinamento sobre a atividade muscular de eqüinos submetidos à prova de resistência. Acta Scientiarum. Animal Science, v.30, n.3, p.307-315, 2008.

BRANDI, R.A.; FURTADO, C.E.; MARTINS, E.N.; FREITAS, E.V.V.; LACERDA-NETO, J.C.; QUEIROZNETO, A. RIBEIRO, L.B. Desempenho de eqüinos submetidos a enduro alimentados com níveis de óleo de soja na dieta. Revista Brasileira de Saúde e Produção Animal [online], v.10, n.2, p.311-321, 2009.

BRANDI, R.A.; FURTADO, C.E.; MARTINS, E.N.; FREITAS, E.V.V.; LACERDA-NETO, J.C.; QUEIROZNETO, A. RIBEIRO, L.B. Parâmetros bioquímicos de eqüinos submetidos à simulação de prova de enduro recebendo dietas com adição de óleo de soja. Revista Brasileira de Zootenia, v.39, n.2, p.313-319, 2010.

EVANS, C. Physiology of equine performance and associated tests of function. Equine Veterinary Journal, v.39, n.4, p.373-383, 2007.

FRAPE, D. Nutrição e Alimentação de Equinos. São Paulo: Roca, 2008. 602p.

GEELEN, S.N.J.; OLDRUITENBORGHOOSTERBAAN, M.M.S.; BEYNEN, A.C. Dietary fat supplementation and equine plasma lipid metabolism.

Equine Veterinary Journal, v.30, p.475-478, 1999. Suppl.

GEOR, R.J.; McCUTCHEON, L.J.; HINCHCLIFF, K.W.; SAMS, R.A. Training-induced alterations in glucose metabolism during moderate-intensity. Equine Veterinary Journal, v.34, p.22-28, 2002. Suppl.

GOBESSO, A.A.O.; MOREIRA, A.M.F.O.; TAMAS, W.T.; RIBEIRO, R.M.; PREZOTTO, L.D.; GONZAGA, I.V.F.; ETCHICHURY, M.; BRANDI, R.A. Digestibilidade aparente e concentrações plasmáticas de triglicerídeos e colesterol em eqüinos alimentados com fontes de óleo vegetal.

Revista Brasileira de Saúde e

Produção Animal [online], v.12, n.1. p.254-263, 2011.

GODOI, F.N.; ALMEIDA, F.Q.; SALIBA, E.O.S.; VENTURA, H.T.; FRANÇA, A.B.; RODRIGUES, L.M. Consumo, cinética digestiva e digestibilidade de nutrientes em eqüinos atletas alimentados com dietas contendo óleo de soja. Revista Brasileira de Zootecnia, v.38, p.1928-1937, 2009a.

GODOI, F.N.; ALMEIDA, F.Q.; GUARIENTI, G.A.; SANTIAGO, J.M.; GUEDES JUNIOR, D.; NOGUEIRA, Y.C.; BRASILEIRO, L.S. Perfil hematológico e características das fezes de eqüinos consumindo dietas hiperlipidêmicas. Ciência Rural, v.39, n.9, p.2571-2577, 2009 b.

GOMIDE, L.M.W.; MARTINS, C.B.; OROZCO, C.A.G.; SAMPAIO, R.C.L.; BELLI, T.; BALDISSERA, V.; LACERDA NETO, J.C. Concentrações sanguíneas de lactato em eqüinos durante a prova de fundo do concurso completo de equitação. Ciência Rural, v.36, n.2, p.509-513, 2006. 
HALLEBEEK, J.M.; BEYNEN, A.C. The plasma level of triacylglycerols in horses fed high-fat diets containing either soybean oil or palm oil. Journal Animal Physiology and Animal Nutrition, v.86, p.111-116, 2002.

HAMBLETON, P.L.; SLADE, L.M.; HAMAR, D.W; KIENHOLZ, E.W.; LEWIS, L.D. Dietary fat and exercise conditioning effect on metabolic parameters in the horse. Journal of Animal Science, v.51, p.1330-1339, 1980.

HARKINS, J.D.; MORRIS, G.S.; TULLEY, R.T.; NELSON, A.G.; KAMERLING, S.G. Effect of added dietary fat on racing performance in thoroughbred horses. Equine

Veterinary Science, v.12, n.2, p.123129, 1992.

HARRIS, P.A.; PAGAN, J.D.; CRANDELL, K.G.; DAVIDSON, N. Effect of feeding thoroughbred horses a high unsaturated or saturated vegetable oil supplemented diet for 6 months following a 10 month fat acclimation.

Equine Veterinary Journal, v.30, p.468-474, 1999. Suppl.

KING, S.S.; ABUGHAZALEH, A.A.; WEBEL, S.K.; JONES, K.L.

Circulating fatty acid profiles in response to three levels of dietary omega-3 fatty acid supplementation in horses. Journal of Animal Science, v.86, p.1114-1123, 2008.

LINDBERG, J.E.; ESSÉN-

GUSTAVSSON, B.; DAHLBORN, K.; GOTTLIEB-VEDI, M.; JANSSON, A. Exrecise response, metabolism at rest and digestibility in athletic horses fed high-fat oats. Equine Veterinary, v.36, p.626-630, 2006. Suppl.
MARQUEZE, A.; KESSLER, A.M.; BERNARDI, M.L. Aumento do nível de óleo em dietas isoenergéticas para cavalos submetidos a exercício. Ciência Rural, v.31, n.3, p.491-496, 2001.

MATTOS, F.; ARAÚJO, K.V.; LEITE, G.G.; GOULART, H.M. Uso do óleo na dieta de eqüinos submetidos ao exercício. Revista Brasileira de Zootecnia, v.35, p.1373-1380, 2006.

NATIONAL RESEARCH COUNCIL NRC. Nutrients requirements of horses. 6.ed. Washington, D.C., 2007. $341 \mathrm{p}$.

MORGADO, E.; GALZERANO, L. Utilização de óleos em dietas para equinos. Revista Eletrônica

Veterinária, v.7, n.10, p.1-14, 2006.

O'CONNOR, C.I.; LAWRENCE, L.M.; LAWRENCE, A.C.St. JANICKI, K.M; WARREN, L.K.; HAYES, S. The effect of dietary fish oil supplementation on exercising horses. Journal of Animal Science, v.82, n.3, p.2978-2984, 2004.

\section{OLDRUITENBORGH-}

OOSTERBAAN, M.M.S.; ANNEE, M.P.; VERDEGAAL, E.J.M.M.; LEMMENS, A.G.; BEYNEN, A.C. Exercise and metabolism-associated blood variables in Standardbreds fed either a low or a high fat diet. Equine Exercise Physiology, v.34, p.29-32, 2002.

OLIVEIRA, R.N.; MARQUES, A.P.; XAVIER, P.R.; ALVES, G.E.S.; PAES, P.R.O.; GOBESSO, A.A.O. Avaliação hematológica e bioquímica de eqüinos suplementados com óleo de arroz semirrefinado, rico em gamaorizanol. Arquivo Brasileiro de Medicina Veterinária e Zootecnia, v.65, n.5, p.1043-1047, 2010. 
PICCIONE, G.; ASSENZA, A.; BORRUSO, M.; FAZIO, F.; CAOLA, G. Daily pattern of some fatty acids in the athletic horse. Journal of Animal Physiology and Nutrition, v.93, p.714, 2009.

RESENDE JÚNIIOR, T.; REZENDE, A.S.C.; LACERDA JÚNIOR, O.V.; BRETAS, M.; LANA, A.; MOURA, R.S.; RESENDE, H.C. Efeito do nível de óleo de milho adicionado á dietas de eqüinos sobre a digestibilidade dos nutrientes. Arquivo Brasileiro de Medicina Veterinária e Zootecnia, v.56, n.1, p.69-73, 2004.

RIBEIRO, W.P.; VALBERG, S.J.; PAGAN, J.D.; ESSEN GUSTAVSSON, B. The effect of varying dietary starch and fat content on serum creatinase kinase activity and substrate availability in equine polysaccharide storage myopathy. Journal Veterinary International Medicine, v.18, n.3, p.887-894, 2004.

RIBEIRO, R.M.R.; PASTORI, W.T.; FAGUNDES, M.H.R.; PREZOTTO, L.D.; GOBESSO, A.A.O. Efeito da inclusão de diferentes fontes lipídicas e óleo mineral na dieta sobre a digestibilidade dos nutrientes e os níveis plasmáticos de gordura em eqüinos.

Revista Brasileira de Zootecnia, v.38, n.10, p.1989-1994, 2009.
SAS Institute. SAS user's: guide statistics. Cary, 2000. 211p.

STUBBS, N.C.; KAISER, L.J.; HAUPTMAN, J.; CLAYTON, H.M. Dynamic mobilization exercises increase cross sectional area of musculus multifidus. Equine Veterinary Journal, v.43, n.5, p.522$529,2011$.

WATANABE, M.J.; THOMASSIAN, A.; TEIXEIRA NETO, F.J.; ALVES, A.L.G.; HUSSNI, C.A.; NICOLETTI, J.L.M. Alterações do pH, da $\mathrm{Po} 2 \mathrm{e}$ da Pco2 arteriais e da concentração de lactato sanguíneo de cavalos da raça Árabe durante exercício em esteira de alta velocidade. Arquivo Brasileiro de Medicina Veterinária e Zootecnia, v.58, n.3, p.320-326, 2006.

Data de recebimento: $28 / 11 / 2012$

Data de aprovação: 07/03/2013 